Mozer de Miranda Ramos ${ }^{1}$ O https://orcid.org/0000-0001-5164-1543

Elder Cerqueira-Santos Ohtps://orid.org/0000-0003-1116-639

\section{Ansiedade social: adaptação e evidências de validade da forma curta da Social Interaction Anxiety Scale e da Social Phobia Scale para o Brasil}

\author{
Social anxiety: adaptation and evidence of validity of the short form of \\ the Social Interaction Anxiety Scale and Social Phobia Scale for Brazil
}

D0I: $10.1590 / 0047-2085000000304$

\begin{abstract}
RESUMO
Objetivo: A ansiedade social é um transtorno psicológico pouco publicizado, mas com importante distribuição epidemiológica. O presente estudo teve como objetivo adaptar para o contexto brasileiro as versões reduzidas da Social Interaction Anxiety Scale (SIAS) e da Social Phobia Scale (SPS) (SIAS-6 e SPS-6) e produzir evidências de validade. Essas escalas avaliam ansiedade em interações sociais e ansiedade social diante da possibilidade de escrutínio por outras pessoas, sendo complementares. Métodos: Como são escalas com reconhecido uso internacional, a adaptação para o Brasil pode trazer contribuições para as investigações clínicas e científicas acerca do transtorno de ansiedade social. Foram realizados processos criteriosos para a adaptação (tradução, avaliação por juízes, retrotradução, avaliação pelo público-alvo) e conduzido um levantamento on-line com 1.049 pessoas, maiores de 18 anos, com média de idade de 25,98 anos (DP =7,55). Resultados: Utilizaram-se Análises Fatoriais Confirmatórias e, desse modo, constatou-se que os índices de ajuste produzidos são, em geral, adequados ( $\mathrm{SRMR}<0,04, \mathrm{CFI}>0,96, \mathrm{TLI}>0,94$ e RMSEA $<0,05$ ) e que os itens possuem cargas fatoriais adequadas (entre 0,441 e 0,837). As evidências de validade discriminante também se mostraram satisfatórias, conseguindo diferenciar grupos por renda e orientação sexual, como esperado pela literatura. Conclusões: Os resultados sugerem que as escalas são adequadas para uso no Brasil, tanto para uso em separado como para uso combinado, como fatores correlacionados.
\end{abstract}

\section{PALAVRAS-CHAVE}

Fobia social, ansiedade, saúde mental, análise fatorial, psicometria.

\begin{abstract}
Objective: Social anxiety is a poorly publicized psychological disorder, but with an important epidemiological distribution. The present study aimed to adapt the reduced versions of the Social Interaction Anxiety Scale (SIAS) and Social Phobia Scale (SPS) (SIAS-6 and SPS-6) to the Brazilian context and produce evidence of validity. These scales assess anxiety in social interactions and social anxiety in face of the possibility of scrutiny by other people, being complementary. Methods: As they are scales with recognized international use, adaptation to Brazil can bring contributions to clinical and scientific investigations about Social Anxiety Disorder. Judicious processes for adaptation were carried out (translation, evaluation by judges, back-translation, evaluation by the target audience) and an online survey was conducted with 1,049 people, over 18 years of age with an average age of 25.98 years (SD = 7.55). Results: Confirmatory Factor Analysis was used and, thus, it was found that the adjustment indexes produced are, generally, adequate (SRMR $<0.04, \mathrm{CFI}>0.96$, TLI $>0.94$ and RMSEA $<0.05$ ) and that the items have adequate factor loads. Evidence of discriminant validity was also satisfactory, managing to differentiate groups by income and sexual orientation as expected by the literature. Conclusions: The results suggest that the scales are suitable for use in Brazil, both for separate use and for combined use, as correlated factors.
\end{abstract}

\section{KEYWORDS}

Social phobia, anxiety, mental health, factor analysis, psychometrics. 


\section{INTRODUÇÃO}

O transtorno de ansiedade social (TAS), ou fobia social, é essencialmente o "medo ou ansiedade acentuados ou intensos de situações sociais nas quais o indivíduo pode ser avaliado pelos outros"1. É insistentemente confundido com timidez ou com outros transtornos de ansiedade². Apesar disso, apresenta uma distribuição epidemiológica relevante nas populações ocidentais e um quadro de desenvolvimento persistente durante a juventude, podendo associar-se a outros transtornos de ansiedade, transtorno depressivo maior ou transtornos associados ao uso de substâncias, comumente antecedendo outros transtornos em caso de comorbidade. Sua prevalência média é estimada entre 2\% e 16\%; no Brasil, a amplitude dos resultados das pesquisas é bastante grande, variando em pesquisas recentes entre 1,8\% e 42,4\%, havendo, de qualquer modo, indícios de larga distribuição sintomatológica na população, em especial nos jovens ${ }^{1,3-9}$. A diversidade de métodos utilizados e a falta de estatísticas oficiais dificultam a avaliação da incidência.

Indivíduos com TAS comumente desejam evitar situações sociais que os possam expor, superdimensionando situações por meio da ansiedade e/ou do medo. Quando ocorre, ou na iminência de ocorrer, experimentam sintomas físicos e/ou emocionais, como sudorese e dificuldade de concentração. A evitação costuma estar relacionada à possibilidade de receberem atenção, se exporem ou serem avaliados. Costumam apresentar baixa autoestima e alta autocrítica-11. O TAS costuma iniciar na infância até a adolescência, não sendo comum o seu surgimento após a juventude. Além disso, costuma afetar mais as mulheres e pode ser mais severo nesse grupo 1,912,13.

No TAS, há uma tendência em interpretar como negativas situações sociais ambíguas e de catastrofizar eventos sociais levemente negativos. Diversos são os prejuízos funcionais que podem decorrer do TAS; eles podem estar relacionados a distanciamento social e prejuízo nas relações sociais ou a dificuldade em desempenhar atividades de trabalho e lazer2,6,14. Desse modo, prejuízos na vida afetiva, financeira e social podem estar relacionados a sintomas de ansiedade social, visto que o processo de desenvolvimento social do indivíduo é afetado. Tudo isso, tem um nítido impacto na saúde psicológica das pessoas com TAS'.

Sob uma perspectiva evolucionária, a ansiedade social tem princípios adaptativos, podendo contribuir para os modos de apresentação e comportamento em sociedade, fatores importantes para a integração social e desejabilidade social. Entretanto, a desregulação desses mecanismos estaria associada à condição de sofrimento que o TAS proporciona, afetando justamente a adaptabilidade do indivíduo nas áreas que, evolutivamente, esses investimentos da ansiedade social visavam contribuir. A posição social e a afiliação são fundamentais para o sucesso dos indivíduos, seja de hominídeos ancestrais em savanas até moradores dos grandes centros atuais. Portanto, investir em modular comportamentos ajudou a promover sucesso social, seja com relação a poder, autoridade e dominação (posição social), seja com relação a segurança, comunhão e moralidade (afiliação). Desse modo, intervenções acerca da ansiedade social deveriam operar no campo da adequação e dimensionamento comportamental, mais do que na extinção de comportamentos que possuem associações epigenéticas e que em dada medida são importantes para a regulação social|15,16.

Há diversos instrumentos para mensurar e rastrear ansiedade social; entre eles, há dois com importante destaque em pesquisas e que não possuem versão adaptada para o Brasil| 17,18: o Social Interaction Anxiety Scale (SIAS, relacionada a medos mais gerais na interação social) e o Social Phobia Scale (SPS, relacionada ao medo associado a realização de atividade cotidianas) ${ }^{19}$. São instrumentos complementares que avaliam facetas associadas, mas distintas, do TAS. Esses instrumentos têm sido utilizados em diversas investigações e adaptados para diferentes contextos culturais, como Suécia, Japão e Espanha ${ }^{20-25}$. Essas escalas possuem 20 itens cada e excelentes indicadores psicométricos e clínicos.

Foram elaboradas versões mais curtas dessas escalas - a SIAS-6 e a SPS-6 ${ }^{26}$ - contando com apenas seis itens cada uma, originalmente no contexto australiano. As versões curtas apresentam resultados bastante satisfatórios em comparação às versões integrais das escalas, apresentando a vantagem de ter um tamanho reduzido, facilitando a aplicação ${ }^{27}$.

O presente estudo objetivou adaptar para o contexto brasileiro as versões reduzidas da SIAS e SPS (SIAS-6 e SPS6) e produzir evidências de validade. É importante que haja variabilidade dos instrumentos utilizados no país e, dada a relevância e os bons indicadores psicométricos, faz-se necessário o processo de adequação das escalas para o Brasil. Além disso, esse processo pode possibilitar o alinhamento de investigações desenvolvidas no Brasil com as internacionais, criando possibilidades comparativas.

\section{MÉTODOS}

As versões reduzidas das escalas SIAS e SPS26 foram denominadas em português de Escala de Ansiedade em Interação Social Reduzida (SIAS-6) e Escala de Ansiedade Social Reduzida (SPS-6). Como estavam originalmente em inglês, cinco etapas criteriosas foram desenvolvidas para adaptação das escalas para o Brasil, seguindo recomendações de Borsa, Damásio e Bandeira28: tradução, avaliação de especialistas, retrotradução, grupo de discussão e estudo empírico. Uma das autoras da versão reduzida, a Dra. Lorna Peters, foi contatada e expressou incentivos para a realização deste trabalho de adaptação das escalas. 
Foi realizado um survey $(n=1.049)$ em uma plataforma on-line, após a aprovação dessa pesquisa pelo Comitê de Ética em Pesquisa da Universidade Federal de Sergipe. Foram observados os princípios éticos que regulamentam a realização de pesquisa com seres humanos, previstos nas Resoluções CNS nos 466/12 e 510/16. Todos os participantes foram devidamente informados dos objetivos e procedimentos da pesquisa por meio de um Termo de Consentimento Livre e Esclarecido. Apenas os que declararam concordar com os termos da pesquisa participaram da resolução do questionário on-line.

\section{Instrumentos e procedimentos}

SIAS-6 e SPS-6: adaptadas de Peters ${ }^{26}$ para o Brasil por este estudo, possuem, cada uma, seis itens ( $a=0,836$ e 0,868), respondidas por uma escala de concordância tipo Likert de cinco pontos. Mini-inventário de Fobia Social29,30: contém três itens $(a=0,81)$, respondidos por uma escala de cinco pontos. Questionário socioidentitário: desenvolvido pelos autores, apresenta questões sobre aspectos como idade, sexualidade, identificação étnico-racial, renda e escolaridade.

Os instrumentos foram organizados em um formulário on-line e divulgados por redes sociais (Instagram, Facebook, Twitter e WhatsApp) para brasileiros, maiores de idade, de todo o país, por 13 dias. Os dados produzidos foram transferidos para o software estatístico JASP, no qual foram desenvolvidas as análises.

\section{Participantes}

A amostra foi direcionada à população geral, não havendo controles acerca das condições sintomatológicas dos participantes. O modelo amostral foi não probilístico, por conveniência e não aleatório. O critério para definição do tamanho da amostra foi pautado pela análise de dados e necessidade de realização de Análise Fatorial, a qual estima que amostras maiores $(n>1.000)$ contribuem para a confiabilidade dos resultados. Participaram deste estudo 1.049 pessoas, entre 18 e 56 anos, com média de idade de 25,98 anos ( $D P=7,55$ ). A maior parte da amostra foi composta por homens $(59,8 \%)$ - mulheres representaram 39,1\%, não binários 1\%, havendo também uma travesti entre os entrevistados. Os estados que mais contribuíram com respondentes foram Sergipe (32,3\%), São Paulo (14,6\%), Bahia (13,6\%) e Rio Grande do Sul (10,8\%). Mais da metade reside na capital de seu estado (55\%), cidades grandes (19,4\%), médias (14\%) e pequenas (11,5\%) do interior também foram representadas. Foram os próprios respondentes que classificaram o tamanho de suas cidades. A divisão da renda foi extraída da classificação do Instituto Brasileiro de Geografia e Estatística, na qual classe A tem renda familiar mensal igual ou superior a 20 salários mínimos, B, entre 10 e 20, C, entre 4 e 10, D, entre 2 e 4, e E, abaixo de 2 salários. Aqui foi considerada uma sexta classificação, a E-, para aqueles com menos de 1 salário mínimo. Os dados da amostra estão especificados na tabela 1.

\section{Análise de dados}

Foram desenvolvidas investigações estatísticas a fim de produzir evidências de validade para a SIAS-6 e a SPS-6 no Brasil. Para tal, foram realizadas Análises Fatoriais Confirmatórias (AFC) para cada uma das escalas, visto que versões reduzidas já apresentam um modelo fatorial predefinido e consistente. Como modelo alternativo, foi realizada a AFC da união das escalas, considerando-as como fatores correlacionados. Além disso, foi realizada a correlação de Pearson entre a SIAS-6, a SPS-6 e a soma dessas duas escalas com a Mini-SPIN, em busca de evidências de validade convergente. Por fim, realizaram-se testes t e ANOVA. A fim de obter indicativos de validade de critério da SIAS-6 e da SPS-6, os grupos investigados estavam agrupados por: orientação sexual, status de relacionamento, renda, identificação étnico-racial e gênero. A consistência interna foi avaliada pelo alfa de Cronbach e pelo ômega de McDonald.

\section{RESULTADOS}

\section{Adaptação da escala}

Os instrumentos foram traduzidos por dois tradutores independentes: um psicólogo fluente em inglês e uma tradutora profissional. A escolha dos tradutores é uma fase importante para a qualidade final da escala, por isso recomendaram-se critério e atenção aos escolhidos. O processo foi feito por e-mail, pelo qual foram passadas as instruções.

As versões traduzidas foram unificadas pelos autores, percebendo-se que o nível de concordância entre as versões havia sido alto. Essa versão foi submetida à análise de três juízes especialistas em psicometria ou clínica psicológica via e-mail, todos com pós-graduação stricto sensu. Os especialistas revisaram a adequação da tradução e propuseram soluções de adequação de itens para o contexto brasileiro - por exemplo, alternativas para "elevadores" e "trens", que não são universais no contexto brasileiro. Outra ponderação solicitada aos juízes foi com relação à inserção do contexto de estudo junto ao de trabalho para ampliar o alcance da escala em um dos itens.

Os especialistas sugeriram o ajuste da tradução de alguns termos que, por terem tradução complexa ou pouco usual, geraram dúvidas e exigiram que os pesquisadores tomassem novas decisões. "Conspicuous" e "might" são exemplos dos termos que levantaram dúvidas, mas que junto aos especialistas pode-se alcançar consenso posteriormente. Foi também nessa fase que um dos especialistas sugeriu que as escalas se tornassem escalas de concordância, mantendo cinco pontos. Após longa discussão e investigação, resolveu-se acatar a sugestão inicialmente e propor a modificação para a autora australiana dessa versão da escala. A principal justificativa é a de que esse formato é mais facilmente entendido no Brasil e mais popularizado, facilitando a aplicação e diminuindo erros no processo. 
Tabela 1. Perfil dos participantes

\begin{tabular}{|c|c|c|c|}
\hline Características & Grupos & Frequência $(n)$ & Porcentagem \\
\hline \multirow[t]{5}{*}{ Orientação sexual } & Heterossexual & 652 & $62,2 \%$ \\
\hline & Homossexual & 217 & $20,7 \%$ \\
\hline & Bissexual & 154 & $14,7 \%$ \\
\hline & Assexual & 7 & $0,7 \%$ \\
\hline & Pansexual & 19 & $1,8 \%$ \\
\hline \multirow[t]{6}{*}{ Identificação étnico-racial } & Branco & 528 & $50,3 \%$ \\
\hline & Preto & 128 & $12,2 \%$ \\
\hline & Pardo & 365 & $34,8 \%$ \\
\hline & Amarelo & 18 & $1,7 \%$ \\
\hline & Indígena & 6 & $0,6 \%$ \\
\hline & Outros & 4 & $0,4 \%$ \\
\hline \multirow[t]{5}{*}{ Relacionamento } & Solteiro(a) & 456 & $43,5 \%$ \\
\hline & Namorando & 343 & $32,7 \%$ \\
\hline & Casado(a) & 165 & $15,7 \%$ \\
\hline & Ficando & 71 & $6,8 \%$ \\
\hline & Outros & 14 & $1,3 \%$ \\
\hline \multirow[t]{6}{*}{ Escolaridade } & Fundamental completo/incompleto & 11 & $1 \%$ \\
\hline & Médio completo/incompleto & 170 & $16,2 \%$ \\
\hline & Superior incompleto & 453 & $43,2 \%$ \\
\hline & Superior completo & 214 & $20,4 \%$ \\
\hline & Pós-graduação lato sensu & 106 & $10,1 \%$ \\
\hline & Pós-graduação stricto sensu & 95 & $9,1 \%$ \\
\hline \multirow[t]{6}{*}{ Renda domiciliar média } & 20 salários mínimos ou mais (A) & 68 & $6,5 \%$ \\
\hline & De 10 a 20 salários mínimos (B) & 110 & $10,5 \%$ \\
\hline & De 4 a 10 salários mínimos (C) & 271 & $25,8 \%$ \\
\hline & De 2 a 4 salários mínimos (D) & 266 & $25,4 \%$ \\
\hline & De 1 a 2 salários mínimos $(E)$ & 224 & $21,4 \%$ \\
\hline & Até 1 salário mínimo (E-) & 110 & $10,5 \%$ \\
\hline \multirow[t]{5}{*}{ Região } & Nordeste & 578 & $55,1 \%$ \\
\hline & Sudeste & 255 & $24,3 \%$ \\
\hline & Sul & 154 & $14,7 \%$ \\
\hline & Norte & 31 & $3,0 \%$ \\
\hline & Centro-Oeste & 26 & $2,5 \%$ \\
\hline
\end{tabular}

A versão pós-interferência dos especialistas foi submetida a retrotradução, para, assim, ser enviada à autora da versão reduzida, que poderia avaliar a manutenção do sentido dos itens. Foram solicitadas versões a dois tradutores independentes: apesar dos dois serem brasileiros, um era residente nos Estados Unidos há mais de uma década e o outro possui certificação avançada na língua. As duas retrotraduções foram encaminhadas à Dra. Lorna Peters, que elogiou as versões e sugeriu dois ajustes finais no processo de tradução, a fim de manter o sentido dos itens. Além disso, a pesquisadora não se opôs à possibilidade de transformar as escalas em itens de concordância estilo Likert.
A partir disso, as versões preliminares das escalas foram enviadas para um grupo de diferentes pessoas (não acadêmicas) que poderiam compor o público-alvo da escala. Os itens foram positivamente avaliados quanto a sua compreensibilidade e fácil interpretação pelo público geral. Assim, encerrou-se a etapa de adaptação focada no conteúdo e forma dos itens e partiu-se para a etapa de produção de outras evidências de validade; para tal, realizou-se um estudo empírico no qual as escalas puderam ser aplicadas para mais de mil respondentes brasileiros. Esses dados foram utilizados para as análises estatísticas e psicométricas presentes neste estudo. 


\section{Produção de evidências psicométricas}

Inicialmente, verificaram-se os valores dos alfas de Cronbach e ômegas de McDonald de cada uma das escalas. A SPS- 6 apresentou um alfa de 0,868 e um ômega de 0,871; a SIAS-6, um alfa de 0,836 e um ômega de 0,842; valores considerados bons. Além disso, verificaram-se os índices de consistência interna para o modelo em que as escalas são utilizadas como fatores correlacionados: alfa de 0,902 e ômega de 0,904. As AFCs foram realizadas com o estimador DWLS (Diagonally Weighted Least Squares), que é adequado para dados do tipo Likert. Utilizaram-se cinco parâmetros de adequação de ajuste para avaliar os modelos: (a) a razão entre o qui-quadrado e os graus de liberdade $\left(\mathrm{X}^{2} / \mathrm{df}\right)$; (b) a raiz quadrada dos resíduos padronizados (SRMR); (c) o índice de adequação de ajuste
(CFI); (d) o índice de Tucker-Lewis (TLI); (e) a raiz quadrada do erro médio ajustada (RMSEA).

A tabela 2 apresenta os índices de ajuste obtidos. Os índices encontrados são considerados adequados tanto para a SIAS-6 quanto para a SPS-6. Adicionalmente, testou-se o modelo no qual as duas escalas funcionam como fatores correlacionados e os índices de ajuste foram igualmente bons.

Considerou-se como parâmetro de ajuste: $\left(x^{2} / \mathrm{df}\right)$ menor do que $5^{31}$; SRMR menor do que 0,0832,33; CFI e TLI maiores do que 0,90 (sendo desejável acima de 0,95) 32,33; RMSEA menor do que 0,08, considerando que, se for maior do que 0,10, há falta de ajuste ${ }^{33}$. As cargas fatoriais, obtidas nas AFCs, e as estatísticas descritivas dos itens estão dispostas na tabela 3. Nenhuma das escalas infringiu os parâmetros utilizados.

Tabela 2. Índices de adequação de ajustes de Análise Fatorial Confirmatória SIAS-6/SPS-6

\begin{tabular}{|c|c|c|c|c|c|c|}
\hline \multirow[b]{2}{*}{ Modelo } & \multicolumn{6}{|c|}{ Índices de Adequação de Ajustes } \\
\hline & $\chi^{2}(\mathrm{df})$ & $\chi^{2 / d f}$ & SRMR & CFI & TLI & $\begin{array}{l}\text { RMSEA } \\
\text { (IC 90\%) }\end{array}$ \\
\hline SIAS-6 (independente) & $17,531(9)$ & $\underline{1,947}$ & $\underline{0,028}$ & $\underline{0,998}$ & $\underline{0,996}$ & $\underline{0,030}(0,006-0,051)$ \\
\hline SPS-6 (independente) & $23,913(9)$ & $\underline{2,657}$ & $\underline{0,032}$ & $\underline{0,997}$ & $\underline{0,995}$ & $\underline{0,040}(0,021-0,060)$ \\
\hline SIAS-6 e SPS-6 como fatores correlacionados & $88,910(53)$ & 1,677 & $\underline{0,032}$ & $\underline{0,997}$ & $\underline{0,997}$ & $\underline{0,025(0,016-0,034)}$ \\
\hline
\end{tabular}

Nota: Em destaque os índices considerados com ajuste adequado.

Tabela 3. Cargas Fatoriais e estatísticas descritivas dos itens das escalas SIAS-6 e SPS-6

\begin{tabular}{|c|c|c|c|c|c|c|}
\hline Itens & $\begin{array}{c}\lambda \text { (como escalas } \\
\text { independentes) } / \lambda \\
\text { (como fatores } \\
\text { correlacionados) }\end{array}$ & $M(D P)$ & $\begin{array}{l}\text { Min- } \\
\text { Máx }\end{array}$ & $\begin{array}{l}\text { Assimetria/ } \\
\text { Curtose }\end{array}$ & $\begin{array}{l}\text { Alfa/Ômega } \\
\text { (com a } \\
\text { retirada do } \\
\text { item) }\end{array}$ & $\begin{array}{l}\text { Correlação } \\
\text { item - total }\end{array}$ \\
\hline \multicolumn{7}{|l|}{ SIAS-6 } \\
\hline 1. Eu tenho dificuldade de manter contato visual com outras pessoas. & 0,899/0,908 & $2,618(1,357)$ & $1-5$ & $0,153 /-1,457$ & $0,813 / 0,820$ & 0,592 \\
\hline $\begin{array}{l}\text { 2. Acho difícil interagir confortavelmente com pessoas com quem estudo } \\
\text { e/ou trabalho. }\end{array}$ & $0,914 / 0,861$ & $2,202(1,272)$ & $1-5$ & $0,693 /-0,871$ & $0,802 / 0,811$ & 0,647 \\
\hline 3. Eu fico tenso(a) se encontro um conhecido na rua. & $0,872 / 0,908$ & $2,316(1,298)$ & $1-5$ & $0,501 /-1,134$ & $0,810 / 0,819$ & 0,607 \\
\hline 4. Sinto-me tenso(a) se fico sozinho(a) com uma pessoa. & $1,013 / 1,028$ & $2,516(1,321)$ & $1-5$ & $0,313 /-1,284$ & 0,793/0,802 & 0,689 \\
\hline 5. Eu tenho dificuldade em falar com outras pessoas. & $1,102 / 1,055$ & $2,359(1,348)$ & $1-5$ & $0,518 /-1,150$ & $0,783 / 0,790$ & 0,732 \\
\hline 6. Eu tenho dificuldade em discordar do ponto de vista de outra pessoa. & $0,573 / 0,627$ & $2,324(1,302)$ & $1-5$ & $0,616 /-0,918$ & $0,848 / 0,850$ & 0,404 \\
\hline Total - SIAS-6 & - & $14,336(5,854)$ & $6-29$ & $0,340 /-0,913$ & - & - \\
\hline \multicolumn{7}{|l|}{ SPS-6 } \\
\hline 1. Eu fico nervoso(a) que pessoas olhem para mim enquanto caminho na rua. & $1,12 / 1,115$ & $2,849(1,476)$ & $1-5$ & $0,093 /-1,457$ & $0,841 / 0,843$ & 0,696 \\
\hline $\begin{array}{l}\text { 2. Fico preocupado(a) se vou ficar trêmulo(a) ou agitado(a) quando outras } \\
\text { pessoas me observam. }\end{array}$ & $1,147 / 1,155$ & $2,673(1,483)$ & $1-5$ & $0,239 /-1,447$ & $0,839 / 0,842$ & 0,708 \\
\hline $\begin{array}{l}\text { 3. Eu ficaria tenso(a) caso tivesse que sentar frente a frente com outras } \\
\text { pessoas no transporte público. }\end{array}$ & $0,963 / 0,957$ & $2,071(1,308)$ & $1-5$ & $0,903 /-0,559$ & $0,844 / 0,849$ & 0,679 \\
\hline 4. Preocupo-me em fazer algo que vai atrair a atenção das pessoas. & $0,819 / 0,865$ & $2,690(1,383)$ & $1-5$ & $0,166 /-1,331$ & $0,866 / 0,868$ & 0,550 \\
\hline $\begin{array}{l}\text { 5. Quando estou em um local fechado com outras pessoas (por exemplo, } \\
\text { uma sala de espera, um elevador...), fico tenso(a) se olham para mim. }\end{array}$ & $1,123 / 1,123$ & $2,444(1,376)$ & $1-5$ & 0,448/-1,202 & $0,831 / 0,836$ & 0,752 \\
\hline $\begin{array}{l}\text { 6. Eu posso me sentir como se estivesse sendo observado(a) quando estou } \\
\text { em uma fila. }\end{array}$ & $0,912 / 0,872$ & $2,230(1,357)$ & $1-5$ & 0,669/-0,959 & $0,854 / 0,857$ & 0,620 \\
\hline Total - SPS-6 & - & $14,958(6,518)$ & $6-30$ & $0,420 /-0,873$ & - & - \\
\hline
\end{tabular}

Nota: Cargas Fatoriais maiores que 1 são possíveis e viáveis quando a variância residual não apresenta carga negativa, indicando grande importância no conjunto de dados ${ }^{34}$. No conjunto em questão, todas as variâncias residuais das cargas representadas foram positivas. 


\section{Evidências de validade de critério}

Foram realizados testes de diferenças de médias, objetivando compreender como a SIAS- 6 e a SPS- 6 discriminam grupos. Também foi gerada uma matriz de correlação entre a SIAS-6, a SPS-6 e o Mini-SPIN, a versão reduzida de uma escala para identificar ansiedade social com largo uso no Brasil. O objetivo foi verificar se havia indícios de validade convergente nas escalas. SIAS-6 e SPS-6 apresentaram correlação significativa e moderada entre si $(r=0,673)$. Em relação ao Mini-SPIN, apresentaram, respectivamente, $r=0,677$ e $r=0,688$, magnitudes consideradas moderadas. A soma da SIAS-6 com a SPS-6 apresentou correlação significativa e moderada com a Mini-SPIN $(r=0,746)$.

Com relação à renda, foi realizada uma ANOVA para verificar se havia diferença significativa entre os grupos em relação a SIAS-6 $[F(5,1043)=4,607 ; p<0,001]$ e a SPS-6 $[F(5,1043)$ $=5,773 ; p<0,001]$. Os grupos com renda menor (E e E-) apresentaram diferença significativa dos grupos com renda maior ( $A$ e B), tanto na SIAS-6 quanto na SPS- 6 - quanto mais pobres, maior a ansiedade social nessas escalas. Mais especificamente, na SIAS-6 a classe E- diferiu significativamente das classes A, B e D, enquanto a classe E, apenas da A. Já na SPS-6, a classe E- diferiu significativamente das classes A, B e $C$, enquanto a classe $E$, das classes $A$ e $B$.

A ANOVA da SIAS-6 $[F(3,1031)=8,560 ; p<0,001]$ e da SPS-6 $[F(3,1031)=12,468 ; p<0,001]$, com relação ao status de relacionamento, encontrou níveis significativamente mais baixos para os casados, em comparação aos solteiros, namorados e ficantes. Isso significaria, a princípio, que não ser casado estaria mais associado à ansiedade social, entretanto essa afirmação pode não ser verdadeira nessa amostra, visto que os casados possuem uma média de idade 10 anos maior que os não casados, sendo essa diferença bastante significativa $[F(3,1031)=124,991 ; p<0,001]$. Possivelmente, a idade seja a variável que mais influencia a ansiedade social, entretanto, fazendo a correlação da SIAS-6 e da SPS-6 com a idade dos participantes, encontrou-se uma associação fraca, apesar de significativa $(r=-0,240$ e $r=-0,264$, respectivamente; $p<0,001$ ).

Diferente do que se esperava, não foram encontradas diferenças significativas da SIAS-6 $[F(5,1043)=0,375 ; p<0,866]$ e da SPS-6 $[F(5,1043)=0,420 ; p<0,835]$ com relação à identificação étnico-racial dos participantes. Já com relação ao gênero, foram encontradas diferenças significativas de forma limítrofe no SIAS-6 ( $t=-1,938 ; p=0,053)$ e de forma mais clara na SPS-6 $(t=-4,839 ; p<0,001)$. Nas duas escalas, mulheres apresentaram médias superiores às dos homens.

Já com relação à orientação sexual, houve diferença significativa entre grupos, tanto na SIAS-6 $[F(2,1020)=4,757$; $p<0,01]$ quanto na SPS-6 $[F(2,1020)=7,938 ; p<0,001]$. Nos dois casos, os heterossexuais apresentaram médias menores, havendo diferenças significativas com relação aos bissexuais na SIAS-6 e com homossexuais e bissexuais na SPS-6.

\section{DISCUSSÃO}

O processo de adaptação das escalas SIAS-6 e SPS-6 foi bastante criterioso e empenhado em manter a precisão característica dos instrumentos. Apesar disso, algumas modificações necessitaram ser feitas, o que não prejudicou os instrumentos, a julgar pelas evidências de validade levantadas. A mudança mais importante foi a transformação das escalas em itens de concordância. Isso facilitou a aplicação e a compreensão dos instrumentos no Brasil, mas não descaracterizou o conteúdo. No item 5 da SPS-6, em vez de referir-se apenas a elevador, decidiu-se, na versão brasileira, utilizar o termo "lugar fechado", dando como exemplos "sala de espera" e "elevador". A intenção principal era ampliar a identificação da população que não acessa a elevadores. Além de muitas cidades horizontais, a desigualdade social do país faz com que haja diferenças nos ambientes frequentados, mesmo numa grande cidade vertical. Por justificativa semelhante, no item 3 adotou-se "transporte público" no lugar de "ônibus ou trem". Já na SIAS-6, é importante destacar a adição do contexto de estudo no item 2. No original, há referência apenas a trabalho, mas, pensando em universitários, por exemplo, tomou-se tal decisão.

As AFCs obtiveram índices de ajuste considerados adequados, mesmo quando se testou o modelo com as escalas funcionando como fatores correlacionados. Esse resultado suporta o uso das escalas de forma individual e de forma combinada, podendo gerar um indicador único. Visto que investigam aspectos diferentes da sintomatologia do TAS e sua correlação é moderada, não indicando, assim, sobreposição das medidas. O estimador utilizado era adequado para o tipo de dado (ordinal), dando maior precisão à análise. Com relação às cargas fatoriais dos itens, apenas o item 6 da SIAS-6 apresentou um valor mais baixo (médio). É possível que discordar do ponto de vista de alguém assuma conotações no contexto cultural brasileiro que estejam associadas não só à ansiedade na interação social. Entretanto, essa hipótese necessitaria de maior investigação.

A SIAS-6 e a SPS-6 são essencialmente escalas de rastreamento. Podem ser utilizadas no contexto clínico e acadêmi$\mathrm{co}$, de forma individual ou combinada, com a vantagem de serem curtas e de fácil aplicação. Podem auxiliar na triagem de grandes grupos e são facilmente adequáveis para pesquisas científicas. As boas e significativas correlações obtidas com o Mini-SPIN sugerem: as medidas são sensíveis no contexto brasileiro e, apesar de investigarem aspectos relacionados ao mesmo constructo, diferem quanto ao conteúdo e ao método de rastreamento. São, portanto, uma boa alternativa para confirmação de investigações ou para substituição de instrumentos. Em contextos de pesquisa, essa possibilidade permite que o participante não responda várias vezes a apenas um instrumento, o que poderia diminuir sua eficácia. As escalas tiveram desempenho adequado quanto à validade de critério, podendo discriminar de forma satisfatória grupos, 
como aponta a literatura, que possuem diferentes incidências de ansiedade social.

As diferenças com relação à renda reforçam a relação existente entre pobreza e transtornos mentais comuns, entre eles, a ansiedade $\mathrm{e}^{35-38}$. A classe econômica pode ser entendida como um estressor que ajuda a explicar tal associação. Nessa amostra, de modo geral, quanto mais pobre, maior a média na SIAS-6 e na SPS-6. As experiências de opressão e restrição provocadas pela pobreza relacionam-se com a ansiedade social e com as habilidades de interação social. Entretanto, a maioria dos estudos nesse campo investiga transtornos mentais comuns ou ansiedade generalizada, ocasionando um déficit de literatura acerca da associação entre ansiedade social e classe social.

Apesar de o resultado do teste realizado aparentemente indicar a relação entre estar casado e ter sinais menores de ansiedade social, a idade parece ser o principal elemento de confusão da análise, visto que o grupo casado tem média de idade 10 anos superior à dos outros grupos. Os mais jovens costumam ser mais acometidos por esse tipo de transtorno, visto que ele se inicia comumente entre a infância e a juventude ${ }^{1,39,40}$. Além disso, como a amostra tem grande participação de universitários, está sujeita a estressores que são pouco comuns em contexto de trabalho mais estáveis e rotineiros.

Não houve relação entre a identificação étnico-racial e as escalas desse estudo. Ampliando-se a lógica do modelo de estresse de minoria ${ }^{41,42}$, relacionando-o não apenas com minorias sexuais, mas também raciais e de gênero, seria justificável que tal relação fosse estabelecida, visto que pessoas negras sofrem diversos estressores durante a sua vida ${ }^{43}$, esta é possivelmente uma das limitações dos resultados encontrados. Neste estudo, mulheres e não heterossexuais apresentaram maior média na SIAS-6 e na SPS-6, ilustrando como o pertencimento a minorias e a contínua exposição a estressores podem relacionar-se com índices mais negativos de saúde mental, em específico a ansiedade social. Essa evidência pode ser relacionada ao modelo de estresse de minoria, mostrando como a exposição contínua a estressores relacionados a estigmas afeta a saúde mental, ao menos no campo da orientação sexual e do gênero. Em específico, com relação às mulheres, há vasta literatura que aponta para uma prevalência maior de TAS do que em homens. Inclusive, em mulheres há uma tendência maior de agravamento do transtorno e comorbidades. Entretanto, por questões culturais e de gênero, homens procuram tratamento para TAS mais do que mulheres ${ }^{1,12,13}$

\section{CONCLUSÕES}

Avaliando os resultados deste presente estudo, pode-se concluir que houve êxito na adaptação da SIAS-6 e da SPS-6 para o contexto brasileiro. Isso é reforçado pelas boas evidências de validade obtidas. Contou com uma amostra de bom tamanho, permitindo a realização das AFCs. Apesar de ela ser predominantemente jovem, é diversa e possui distribuição geográfica, social e identitária plausível.

Apesar de ser um método válido, a produção on-line dos dados ainda está ligada a uma amostra jovem e escolarizada, e essa é uma das limitações deste estudo. Outra limitação é a não realização de aplicação com grupos clínicos objetivando verificar a eficácia de rastreamento numa amostra brasileira. E essa é a principal indicação para a agenda de investigações propostas a partir deste estudo. Além disso, poderiam ser utilizados outros instrumentos para avaliar a validade convergente e divergente da escala. É preciso que essas associações sejam tratadas em outros estudos.

As evidências obtidas neste estudo incentivam o uso das escalas no Brasil, de forma independente ou combinada, e o aprofundamento das pesquisas. A ansiedade social ainda é carente de investigações em diferentes contextos, principalmente considerando os grupos minoritários. A SIAS- 6 e a SPS-6 discriminaram de forma satisfatória grupos minoritários de gênero e orientações sexual. O mesmo não ocorreu com a identificação étnico-racial, mas são necessárias outras amostras para haver conclusões a esse respeito. Além disso, é urgente desenvolver investigações sobre o ponto de corte das escalas no Brasil, a fim de facilitar e padronizar sua interpretação. Por possuir um tamanho reduzido e ser de fácil aplicação, seu uso pode ser explorado na clínica e nas pesquisas.

\section{CONTRIBUIÇÕES INDIVIDUAIS}

Mozer de Miranda Ramos - Concepção e desenho do estudo e das análises, escrita do manuscrito e aprovação da versão final a ser publicada.

Elder Cerqueira-Santos - Colaboração significativa na concepção e desenho do estudo e na interpretação dos dados; contribuição substancial na revisão crítica do conteúdo intelectual do artigo e aprovação da versão final a ser publicada.

\section{CONFLITO DE INTERESSES}

Os autores declaram não haver conflito de interesses na produção deste estudo.

\section{AGRADECIMENTOS}

À Dra. Lorna Peters, professora da Macquarie University em Sydney, pela disponibilidade e colaboração na elaboração desta pesquisa. 
À Capes, pelo financiamento do projeto por meio do fornecimento de bolsa de estudos.

\section{REFERÊNCIAS}

1. American Psychiatric Association. DSM-5: Manual diagnóstico e estatístico de transtornos mentais. Porto Alegre: Artmed; 2014.

2. Karasewich TA, Kuhlmeier VA. Trait social anxiety as a conditional adaptation: A developmental and evolutionary framework. Develop Rev. 2020;55:100886.

3. Baptista CA, Loureiro SR, de Lima Osório F, Zuardi AW, Magalhães PV, Kapczinski F, et al. Social phobia in Brazilian university students: Prevalence, under-recognition and academic impairment in women. J Affect Disord. 2012;136:857-61.

4. Jefferies P, Ungar M. Social anxiety in young people: A prevalence study in seven countries. PLoS One. 2020;15:e0239133.

5. Machado MB, Ignácio ZM, Jornada LK, Réus GZ, Abelaira HM, Arent CO, et al. Prevalência de transtornos ansiosos e algumas comorbidades em idosos: um estudo de base populacional. J Bras Psiquiatr. 2016;65:28-35.

6. Morais LVD, Crippa JAS, Loureiro SR. Os prejuízos funcionais de pessoas com transtorno de ansiedade social: uma revisão. Rev Psiquiatr Rio Gd Sul. 2008;30:1-13.

7. Orellana JDY, Ribeiro MRC, Barbieri MA, Saraiva MDC, Cardoso VC, Bettiol H, et al. Transtornos mentais em adolescentes, jovens e adultos do consórcio de coortes de nascimento brasileiras RPS (Ribeirão Preto, Pelotas e São Luís). Cad Saúde Pública. 2020;36:e00154319.

8. Regis JMO, Ramos-Cerqueira ATA, Lima MCP, Torres AR. Social anxiety symptoms and body image dissatisfaction in medical students: prevalence and correlates. J Bras Psiquiatr. 2018:67:65-73

9. Stein MB, Stein DJ. Social anxiety disorder. Lancet. 2008;371:1115-25.

10. Burato KRDS, Crippa JADS, Loureiro SR. Transtorno de ansiedade social e comportamentos de evitação e de segurança: uma revisão sistemática. Estud Psicol (Natal). 2009;14:167-74.

11. Cox BJ, Fleet C, Stein MB. Self-criticism and social phobia in the US national comorbidity survey. J Affect Disord. 2004;82:227-34.

12. Asher M, Aderka IM. Gender differences in social anxiety disorder. J Clin Psychol 2018:74:1730-41.

13. Asher M, Asnaani A, Aderka IM. Gender differences in social anxiety disorder: A review. Clin Psychol Rev. 2017:56:1-12

14. Stopa L, Clark DM. Social phobia and interpretation of social events. Behav Res Ther. 2000;38:273-83.

15. Gilbert P. Evolution and social anxiety: The role of attraction, social competition, and social hierarchies. Psychiatr Clin. 2001;24:723-51.

16. Gilboa-Schechtman E, Shachar I, Helpman L. Evolutionary Perspective on Social Anxiety. In: Hofmann SG, DiBartolo PM, eds. Social anxiety: Clinical, developmental, and social perspectives. Waltham, MA: Elsevier Academic Press; 2014. p. 599-622.

17. Osório FDL, Crippa JADS, Loureiro SR. Instrumentos de avaliação do transtorno de ansiedade social. Arch Clin Psychiatry (São Paulo). 2005;32:73-83.

18. Wong QJ, Gregory B, McLellan LF. A review of scales to measure social anxiety disorder in clinical and epidemiological studies. Curr Psychiatry Rep. 2016;18:38.

19. Mattick RP, Clarke JC. Development and validation of measures of social phobia scrutiny fear and social interaction anxiety. Behav Res Ther. 1998;36:455-70.

20. Kanai Y, Sasakawa S, Chen J, Suzuki S, Shimada H, Sakano Y. Development and validation of the Japanese version of social phobia scale and social interaction anxiety scale. Jpn J Psychosom Med. 2004:44:841-50.
21. Mörtberg E, Reuterskiöld L, Tillfors M, Furmark T, Öst LG. Factor solutions of the Social Phobia Scale (SPS) and the Social Interaction Anxiety Scale (SIAS) in a Swedish population. Cogn Behav Ther. 2017:46:300-14

22. Olivares J, García-López LJ, Hidalgo MD. The social phobia scale and the social interaction anxiety scale: factor structure and reliability in a Spanish-speaking population. J Psychoeduc Assess. 2001;19:69-80.

23. Smeltere L, Kuznecovs V, Erts R. Depression and social phobia in essential tremor and Parkinson's disease. Brain Behav. 2017;7:e00781.

24. Villarosa-Hurlocker MC, Madson MB. A latent profile analysis of social anxiety and alcohol use among college students. Addict Behav. 2020;104:106284.

25. Wong QJ, Chen J, Gregory B, Baillie AJ, Nagata T, Furukawa TA, et al. Measurement equivalence of the Social Interaction Anxiety Scale (SIAS) and Social Phobia Scale (SPS) across individuals with social anxiety disorder from Japanese and Australian sociocultural contexts. J Affect Disord. 2019;243:165-74.

26. Peters L, Sunderland M, Andrews G, Rapee RM, Mattick RP. Development of a short form Social Interaction Anxiety (SIAS) and Social Phobia Scale (SPS) using nonparametric item response theory: The SIAS-6 and the SPS-6. Psychol Assess. 2012;24:66.

27. Sunderland M, Afzali MH, Batterham PJ, Calear AL, Carragher N, Hobbs M, et al. Comparing Scores From Full Length, Short Form, and Adaptive Tests of the Social Interaction Anxiety and Social Phobia Scales. Assessment. 2020;27:518-32.

28. Borsa JC, Damásio BF, Bandeira DR. Adaptação e validação de instrumentos psicológicos entre culturas: Algumas considerações. Paidéia (Ribeirão Preto). 2012;22:423-32.

29. D'El Rey GJF, Lacava JPL, Cardoso R. Consistência interna da versão em português do MiniInventário de Fobia Social (Mini-SPIN). Arch Clin Psychiatry (São Paulo). 2007;34:266-9.

30. D'El Rey GJF, Matos CW. Validação da versão em português do Mini-Inventário de Fobia Social (Mini-SPIN). Ciênc Saúde Coletiva. 2009;14:1681-6.

31. Wheaton B, Muthen B, Alwin DF, Summers G. Assessing Reliability and Stability in Panel Models. Sociological Methodology. 1977;8:84-136.

32. Brown TA. Confirmatory Factor Analysis for Applied Research. New York: The Guilford Press; 2006

33. Schreiber JB, Stage FK, King J, Nora A, Barlow EA. Reporting structural equation modeling and confirmatory factor analysis results: A review. J Educ Res. 2006:99:324-37.

34. Matos DAS, Rodrigues EC. Análise Fatorial. Brasília: ENAP. 2019. Disponível em: https:// repositorio.enap.gov.br/bitstream/1/4790/1/Livro\%20An\%C3\%A1lise\%20Fatorial.pdf

35. Alves AAM, Rodriques NFR. Determinantes sociais e económicos da Saúde Mental. Rev Port Saúde Pública. 2010;28:127-31.

36. La Rosa J. Ansiedade, sexo, nível sócio-econômico e ordem de nascimento. Psicol Reflex Crít. 1998;11:59-70.

37. Li C, Wu Q, Liang Z. Effect of poverty on mental health of children in rural China: the mediating role of social capital. Appl Res Qual Life. 2019;14:131-53.

38. Silva DF, Santana PR. Transtornos mentais e pobreza no Brasil: uma revisão sistemática. Tempus Actas de Saúde Coletiva. 2012;6:175-85.

39. Beesdo-Baum K, Bittner A, Pine DS, Stein MB, Höfler M, Lieb R, et al. Incidence of social anxiety disorder and the consistent risk for secondary depression in the first three decades of life. Arch Gen Psychiatry. 2007;64:903-12.

40. Wittchen HU, Fehm L. Epidemiology and natural course of social fears and social phobia. Acta Psychiatr Scand. 2003;108:4-18.

41. Frost DM, Lehavot K, Meyer IH. Minority stress and physical health among sexual minority individuals. J Behav Med. 2015:38:1-8.

42. Meyer IH. Minority stress and mental health in gay men. J Health Soc Behav. 1995;36:38-56.

43. Lima ME0. 0 que há de novo no "novo" racismo do Brasil? Revista Ensaios e Pesquisas em Educação e Cultura. 2019:4:157-77. 